\title{
Multi Sensor System for Analyzing the Thigh Movement during Walking
}

\author{
M. Borghetti, M. Serpelloni, Member, IEEE, E. Sardini, Member, IEEE, Oscar Casas, Member, IEEE
}

\begin{abstract}
Body movements monitoring may allow preventing, diagnosing, and recovering several wrong attitudes that could lead to possible future diseases. A multi sensor system was developed to measure thigh movements in free-living environments all-day long especially during walking. The device is a very simple and portable system based on low-cost technology. It is composed of an inertial sensor and a strain sensor for detecting thigh movements, and of a microcontroller and a Bluetooth module. The measurement data are collected and sent wirelessly to a PC for storing and further analyses. We validated and tested the wearable system on 10 healthy subjects during walking and running by using an accurate inertial motion capture (Mo-cap) system. The inclination of the thigh measured by the proposed system has differed of maximum $5.5^{\circ}$ with respect to the flexion/extension measured by the Mo-cap system. Furthermore, the experimental results have showed that the strain sensor output can also be related to the muscle activity during walking. This assumption has further been sustained when the system is tested during running. Thanks to its high wearability, wireless communication over long distances and long-battery life, the system could be adopted for domestic applications.
\end{abstract}

Index Terms-strain sensor, inertial sensor, wearable sensor, wearable electronic device.

\section{INTRODUCTION}

$\mathrm{N}$ OWADAYS, the interest in the wearable devices for detecting and evaluating the functional ability is increasing. Walking is one the most important activities of the daily life and it usually represents one of the main validation methods to prevent, diagnose, and recovery disabilities and diseases $[1,2]$.

Different measurement systems can be used for the assessment of kinematic and kinetic parameters during walking. The typical equipment adopted in the laboratory includes marker-based optical and video processing systems, force sensing walkways and electromyography [3]. Besides its high performance and accuracy, this equipment is characterized by high costs, high complexity and technological constrains. In free-living environments, wearable devices instead represent an interesting solution since they can be easily adopted during daily life activities. Indeed, they are usually based on wireless and low-cost technology, they do not need complex and heavy equipment; thus, they do not require specific environments. For example, Jensen et al. [4] proposed a swimming exercise tracker, a wearable system for aquatic sports and healthcare. The interest in the inertial motion sensors for healthcare applications has been recently growing. These devices can include accelerometers, gyroscopes and magnetometers. If they are correctly placed on the different body segments, these devices allow tracking the angle and the position of the joints in the space [5], detecting dangerous situations as the freezing of gait in the case of Parkinsonian patients [6]. Despite their high accuracy, the network of several inertial sensors could be very expensive, because it requires complex interfaces, fusion algorithms and protocols for handling it [7]. For example, the protocol includes the modelling of the body, the positioning of the sensors and the algorithm to extract the kinetic parameters. The calibration and the positioning procedure usually is timeconsuming and complex. It includes the alignment of the segment anatomical coordinate system (CS) with the inertial CS. For example, Cutti et. al [8] proposed a method to overcome these problems, obtaining a root mean square error lower than $2.5^{\circ}$ for hip and knee angles. Other fusion algorithms have recently been proposed but they have not been extensively documented. For example, Lebel et al. [9] studied the accuracy over time of Attitude and Heading Reference Systems. They implemented this algorithm in three different commercial inertial systems and they found an average absolute accuracy of $5^{\circ}$. Finally, the inertial sensors are also affected by drifts and noise disturbances.

The recent development in electronic textiles has allowed developing new wearable sensors able to monitor the angle of the body joints [10-14]. These sensors are usually adopted as goniometers. For example, Tesconi et al. [10] proposed a leotard equipped with six sensors for measuring the flexion and extension of knee and hip. These sensors are based on a conductive ink (graphite and silicon rubber) printed directly over the textile. The electrical resistance directly depends on the bending. In this case, the garment design depends on the body dimensions and it influences the sensor response. If this sensing garment is appealing for sport applications, it is less suitable for clinical applications. Mengüç et al. [11] proposed soft strain sensors, one for each joint of the lower limbs. In this case,
M. Borghetti, M. Serpelloni, E. Sardini are with the Department of Information Engineering, University of Brescia, Brescia 25121, Italy (e-mail: m.borghetti001@unibs.it).
Oscar Casas is with the Instrumentation, Sensors and Interfaces Group, Universitat Politècnica de Catalunya, BarcelonaTech, Barcelona 08860, Spain (e-mail: jaime.oscar.casas@upc.edu). 
generic running tights are modified with hook-and-loop fasteners, where the strain sensors are anchored. The resistance changes linearly with the joint angle. Also in this case, the garment is not suitable for medical applications. The textile materials are affected by drift since they change their mechanical properties over time, and a periodical calibration procedure is required. For this reason, Tognetti et. al [13] proposed a wearable system combining e-textiles and low-cost inertial sensing. They tried to fuse the information from these two sensors in order to compensate their drawbacks and measure the knee flexion and extension.

In this paper, we proposed a new multi sensor system for measuring thigh movements all-day long in free-living environments. For example, measuring the range of motion (ROM) of the hip allows detecting abnormal postures or quantifying patient's inabilities; for example, Lewis et al. [15] showed that walking in a forward flexed posture leads to increase the hip flexion of $20^{\circ}$, whereas walking in a swayback posture reduces the hip flexion of more than $5^{\circ}$. The monitoring of the hip flexion is useful also in the case of unilateral transfemoral amputees [16] since the musculoskeletal system changes alter the gait pattern: the duration of the single phases of the gait cycle changes as well as the joint angles of the sound and prosthetic limb [17]. Here the hip plays an important role for transmitting the forces able to move the prosthesis, and a wrong mechanical adaptation of the prosthesis could increase the energy expenditure during locomotion and consequently the risk of cardiac stroke [18]. The skin deformation changes depending on the lower-limb movement and its measurement can give an indication of the muscle activity. Several researches $[19,20]$ provided a detailed skin deformation mapping only during the knee flexion. In fact, the measurement of the skin deformation is quite complex since contraction and extension of the skin is not the same along the same body segment. For all those reasons, we proposed a new wearable system with one strain sensor and one inertial sensor. It does not require a complex calibration procedure and a heavy algorithm implemented on the microcontroller. This system is unobtrusive and portable, due to the long-life battery and the wireless transmission of the data to a laptop equipped with Bluetooth module. However, by developing a custom application, any device with Bluetooth can be used for receiving data, such as smartphone and tablet, allowing continuous and real-time monitoring. Although the Bluetooth transmission does not guarantee low-power consumption, this wearable system is able to monitor continuously the patient for a couple of days. With respect to other products like sensing clothing, this system can be easily worn on subjects with disabilities since the sensors are positioned on elastic straps. The proposed wearable system can be worn over any clothing, and, for this reason, it can be adopted during physical activity or during the rehabilitation therapy at home. The system was tested on ten healthy subjects during walking and running by comparing the results with the ones acquired by an accurate inertial motion capture system. The easiness, good versatility and sufficient low-power consumption allow adopting the proposed system as a tool for recording the able-body people's performance or for

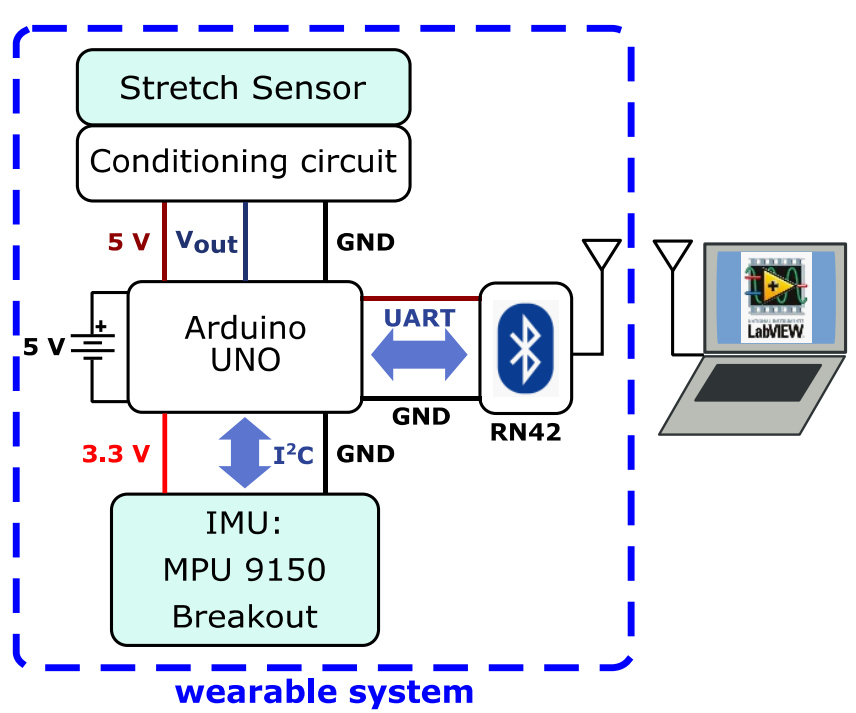

Fig. 1. Block Diagram describing the measurement system. The wearable system includes sensors, conditioning circuits, the microcontroller, the transmitting unit and the power supply. The PC collects the measurements data via Bluetooth connection.

continuously monitoring patients' disabilities in several environments and situations.

\section{SYSTEM DESCRIPTION}

The system consists of two parts: (1) a wearable system, which includes sensors, signal conditioning circuits, a

a)
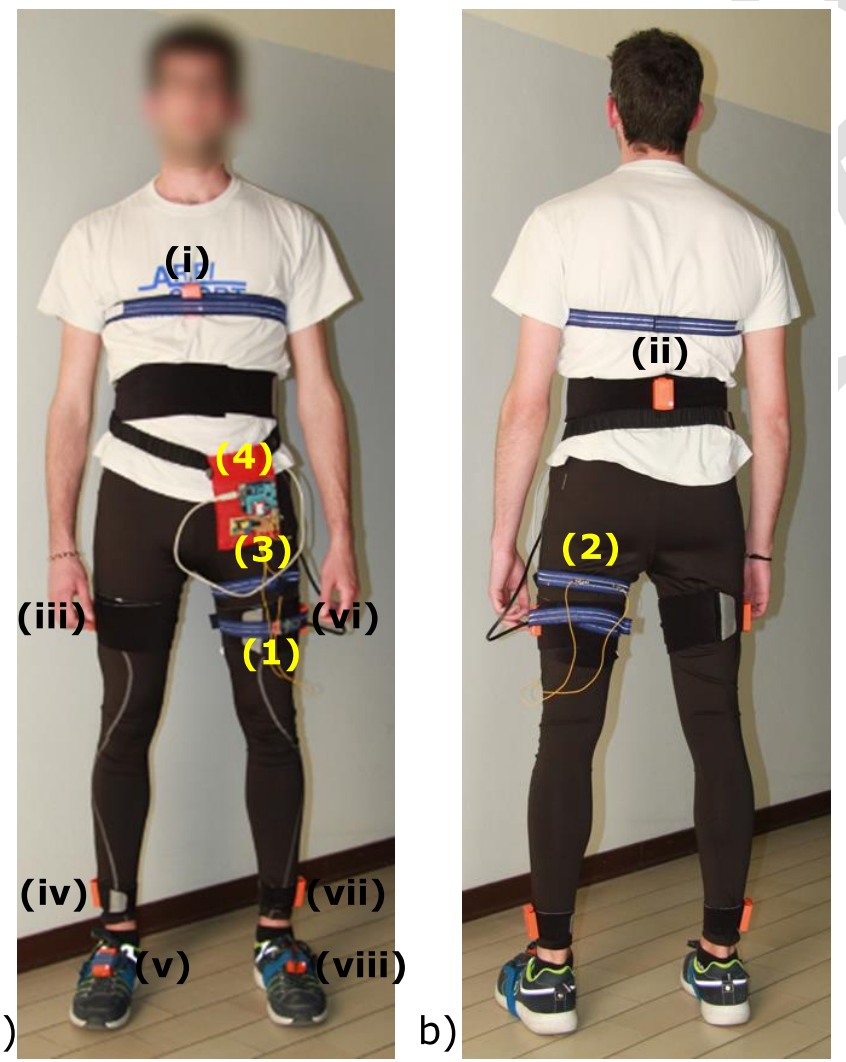

Fig. 2. a) Front and b) back view of the subject wearing the wearable system and the validation system (XSens). The wearable system includes (1) MPU, (2) Stretch Sensor, (3) the electronics, and (4) the battery. The validation system consists of eight IMUs labelled from (i) to (viii). 
microcontroller and a transceiver, and (2) a personal computer (PC) for collecting and elaborating data.

As shown in the block diagram of Fig. 1 and in Fig. 2, the wearable system mainly consists of four parts: (1) one inertial sensor, (2) one strain sensor, (3) the custom electronics for conditioning and transmitting the sensor signals, and (4) a battery.

The MPU 9150 (Fig. 3a) manufactured by InvenSense is used here as Inertial Measurement Unit (IMU). It is a 9-axis MotionTracking MEMS device consisting of a 3-axis accelerometer, a 3-axis gyroscope and a 3-axis digital magnetometer. Accelerometer and gyroscope have 16-bit resolution, whereas the magnetometer has 13-bit resolution. Furthermore, it includes a temperature sensor for drift compensation of the sensors. A 6-axis MotionFusion algorithm is implemented for computing the results in terms of quaternions. In this way, the on-chip processing of the collected data reduces the computational requirements for the system processor, as well as the need for frequent polling of the motion sensor output, thanks to the 1024-bit FIFO buffer.

The breakout board (MPU) commercialized by Sparkfun is adopted for this application due to its small size $(27.94 \mathrm{~mm} \mathrm{x}$ $15.24 \mathrm{~mm} \times 1.60 \mathrm{~mm}$ ). It includes the inertial sensor and the conditioning circuit for interfacing with an external microcontroller through Inter Integrated Circuit $\left(\mathrm{I}^{2} \mathrm{C}\right)$ protocol at $400 \mathrm{kHz}$. The board was firmly fixed to an elastic Velcro strap to tie around the thigh for tracking the leg kinematic.

Our goal is to measure the flexion and the extension of the hip by measuring the angle between the vertical and the thigh segment. The coordinate system of the MPU (local coordinate system) is aligned with the physiological axes of the thigh, while the misalignment with the global coordinate system (reference coordinate system) is estimated before using. Indeed, before the tests, the subject was asked to keep the upright position for ten seconds while the corresponding quaternion was acquired. For each step, we calculated the best-fit plane for the $\mathrm{z}$-axis vector of the local system. Consequently, we could consider the calculated plane as the sagittal plane of the thigh and thus the movements within this plane as the flexion and

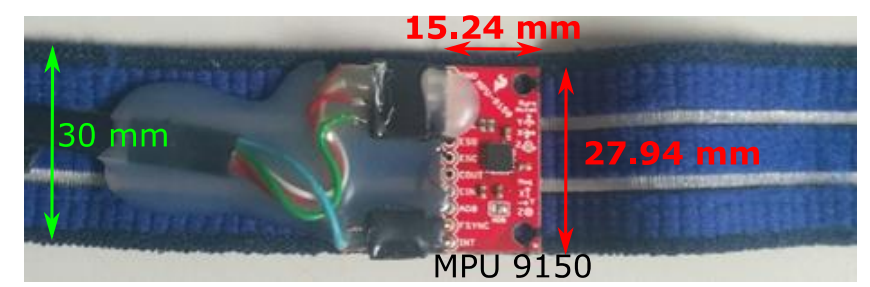

a)

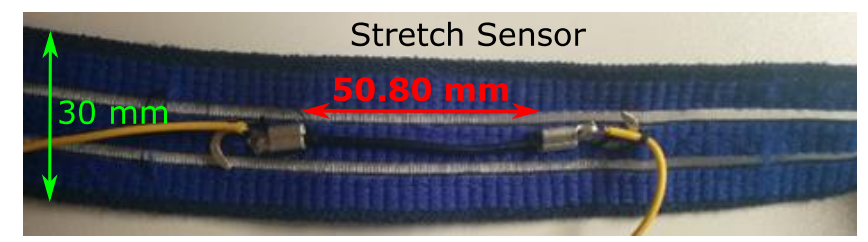

b)

Fig. 3. Sensors adopted in the wearable system: a) inertial system (MPU 9150) fixed on an elastic Velcro strap; b) Strecth Sensor fixed on an elastic Velcro strap. extension of the hip. In this case, the flexion and extension $\left(\alpha_{\mathrm{MPU}}\right)$ corresponds to the angle between the projected z-axis onto the plane and the vertical position.

Stretch Sensor (50.8 $\mathrm{mm}$ long) manufactured by Images Scientific Instruments was used as strain sensor (Fig. 3b) for measuring the thigh movements. It consists of a conductive flexible cord ( $1.78 \mathrm{~mm}$ in diameter). The nominal sensor resistance (zero strain) is near $700 \Omega$ and its electrical resistance increases when the sensor is stretched. In this work, the electrical terminals were fixed on another elastic Velcro strap to be tied around the leg. The sensor was put on the upper posterior thigh, approximately over the biceps femoris, as shown in Fig. 2b. This positioning guarantees the highest response of the sensor and ensures good comfort (see section IVB). The muscle activity during the movement induces the deformation of the skin that induces the deformation of the strap and therefore of the stretch sensor. As confirmed by the experimental results, its resistance $(R)$ can be expressed as a function of the its elongation $(\Delta L)$ by the following formula

$$
R=R_{0}\left(1+G F \cdot \frac{\Delta L}{L_{0}}\right)
$$

where $R_{0}$ and $L_{0}$ are the resistance and the length of the sensor, respectively, when the sensor is not deformed; $G F$ is the gauge factor of the sensor.

A custom electronic board was designed in order to acquire the sensor signal. The board (the schematic is shown in Fig. 4) operates at $5 \mathrm{~V}$.

The sensor resistance is compared to a variable resistor $\left(R_{0}\right)$ with the same value of the nominal sensor resistance through an amplifier. The resulting signal is amplified and shifted for making the output compatible with the microcontroller input (0$5 \mathrm{~V})$. Since the nominal sensor resistance depends on several parameters (tolerance of the production process and environment conditions), we regulated $R_{0}$, which is a trimmer resistor, to a value that permitted to have $0 \mathrm{~V}$ at the output of the board $\left(V_{\text {out }}\right)$ at zero strain before use. The two networks $R_{2} C_{1}$ and $C_{2}$ were introduced as low-pass filter for the sensor response and $\mathrm{V}_{\mathrm{cc}} / 2$. The cut-off frequency is set about to 20.5 $\mathrm{Hz}$ (about five times lower than the sample rate of the acquisition).

The analog output $\left(V_{\text {out }}\right)$ is acquired by Arduino UNO

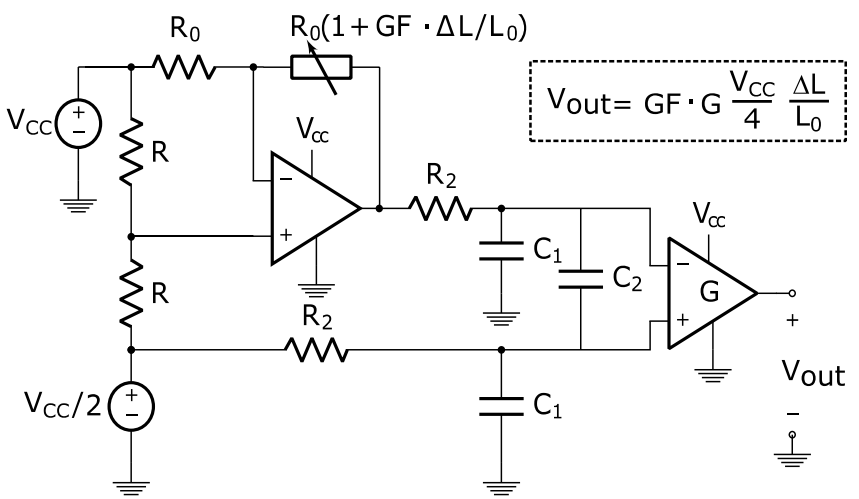

Fig. 4. Simplified schematic of the conditioning circuit for the stretch sensor 
microcontroller, which initializes and collects the data from the inertial system, reads the analog signal of the stretch sensor and sends the elaborated data via Bluetooth to a PC every $10 \mathrm{~ms}$. The selected sample rate is currently used for several gait analysis systems [21]. The analog inputs of Arduino are internally connected to 10-bit analog-to-digital converters. Arduino UNO communicates wirelessly by using a Bluetooth module (Microchip RN42) and the Xbee Shield adapter. The transmission distance is up to $50 \mathrm{~m}$ indoor.

A power bank (Easyacc PB3000M) is the power supply of the entire system. It consists of a $3000 \mathrm{mAh}$ rechargeable Li-ion battery pack and a circuit for powering the entire wearable system at $5 \mathrm{~V}$ and for recharging the battery. Its weight (only $77 \mathrm{~g})$ and its small size $\left(94 \times 23 \times 23 \mathrm{~mm}^{3}\right)$ allow integrating circuit boards and battery pack inside a pocket created over the belt. The maximum power consumption of the entire wearable system is $340 \mathrm{~mW}$. The current consumptions of different parts of the system are reported in Table I.

\section{TABLE I}

\begin{tabular}{l|l}
\multicolumn{2}{l|}{ CURRENT CONSUMPTION OF EACH PART OF THE WEARABLE SYSTEM } \\
\hline Part & \multicolumn{1}{|c}{ Current (mA) } \\
\hline Arduino UNO & 20 \\
MPU-9150 & 4 \\
Stretch Sensor + circuit & 2 \\
Bluetooth module + Shield & 42 \\
\hline
\end{tabular}

As expected, the Bluetooth module is the highest power consumption component.

A LabVIEW Virtual Instruments (VI) was specifically designed for collecting, elaborating, displaying and storing the measurement data transmitted by Arduino and it was executed on a PC equipped with a Bluetooth module. To meet the requirement of a continuous and real time monitoring any portable device equipped with a Bluetooth module, such as smartphones and tablets, could be equally adopted by developing the proper application.

\section{STRETCH SENSOR TESTS}

We tested the stretch sensor to define its mechanical and

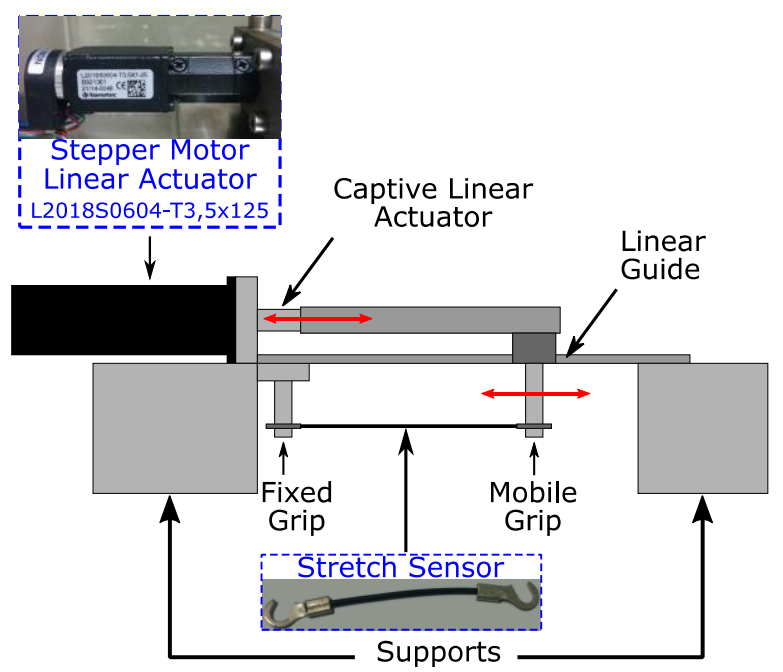

Fig. 5. Schematization of the set-up for determining the relationship between the elongation and the electrical resistance. electrical behavior. We also characterized the ability of the stretch sensor to detect the thigh skin deformation and thus the hip joint angle in the sagittal plane of ten healthy participants (section IV).

For the characterization of the stretch sensors, we carried out three tests: (1) strain ramp test, (2) cyclic loading-unloading test, (3) maintained strain levels for prolonged time and (4) temperature test. The mechanical structure adopted for the first three tests was specially created and it is shown in Fig. 5.

The linear motor moves the mobile grip and imposes the elongation of the sensor via PC, while a $6 \frac{1}{2}$ digits multimeter (Agilent 34401A) measures the electrical resistance. In the first test, the engine moved the mobile grip at constant speed $(0.15$ $\mathrm{mm} / \mathrm{s}$ ) until $20 \%$ of elongation of the sensor. We chose this value since the expected skin deformation during walking is less than $15 \%$, as demonstrated by the validation results. For the same reason, in the second test the sensor was subjected to 60 cycles for $10 \%$ of maximum strain. The speed was set to 2.5 $\mathrm{mm} / \mathrm{s}$. In the third test, the same level of strain was applied and maintained for ten minutes.

For the last test, the sensor was placed unloaded inside a climatic chamber (Perani UC 150/70) and the electrical resistance was recorded by a $61 / 2$ digits multimeter (Agilent 34401A). The temperature was increased gradually and slowly from $10{ }^{\circ} \mathrm{C}$ to $40^{\circ} \mathrm{C}$. A Pt1000 was placed near the sensor and its resistance was measured by another $61 / 2$ digits multimeter (Agilent 34401A). The two multimeters were connected to a PC by a General-Purpose Interface Bus (GPIB), IEEE 488. The whole measurement procedure was controlled by an ownwritten VI.

The results (Fig. 6) of the strain ramp test on the stretch sensor allowed defining the relationship between the resistance and the applied strain. In strain range from $0 \%$ and $15 \%$, the resistance changes $\left(\Delta R / R_{0}\right)$ linearly with the applied strain $(\varepsilon)$. The gauge factor $(G F)$ represents the slope of the linear section and it is defined by the following formula

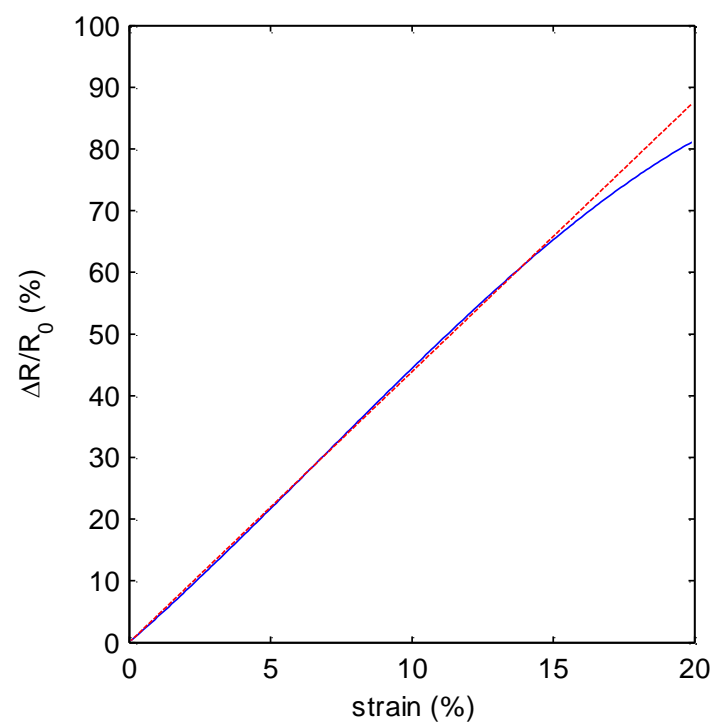

Fig. 6. Normalized resistance change $\left(\mathrm{R}_{0}=858 \Omega\right)$ as a function of the applied strain. The dashed line is the least square regression line calculated in the strain range from $0 \%$ to $15 \%$. The line slope is 4.73 and represents the gauge factor of the sensor. 


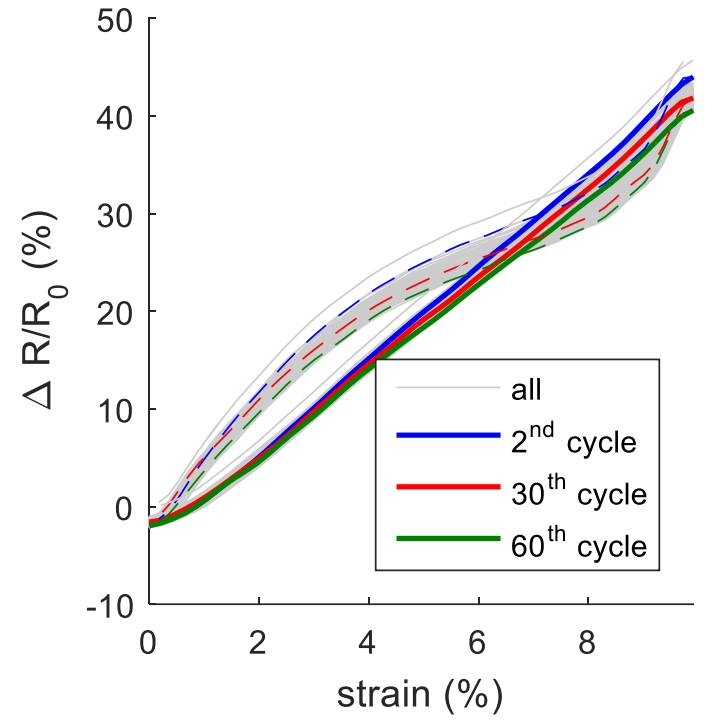

Fig. 7. Response of the stretch sensor under the application of loadingunloading cycles at $10 \%$ of strain; grey curves represent the continuous reading of the sensor on all cycles and the curves corresponding to the $2^{\text {nd }}, 30^{\text {th }}$ and $60^{\text {th }}$ cycle are highlighted. The thicker lines refer the resistance change for positive strain rate (loading section) while the dashed lines refer to the resistance change for negative strain rate (unloading section).

$$
G F=\frac{\frac{R-R_{0}}{R_{0}}}{\frac{L-L_{0}}{L_{0}}}=\frac{\frac{\Delta R}{R_{0}}}{\varepsilon}
$$

where $R$ and $L$ are respectively the resistance and the length of the sensor when a strain $\left(\varepsilon=\Delta L / L_{0}\right)$ is applied, while $R_{0}$ and $L_{0}$ are respectively the resistance and the length of the unstrained sensor.

The gauge factor $G F$ calculated from the results of the strain ramp test (Fig. 6) is 4.73 and this value was used in section IV for calculating the strain $\varepsilon$ of the sensor, starting from the measurements of the $V_{\text {out }}$ and using the formula reported on Fig. 4.

The results of the cyclic loading-unloading test (Fig. 7) define the relationship between the resistance and the strain,

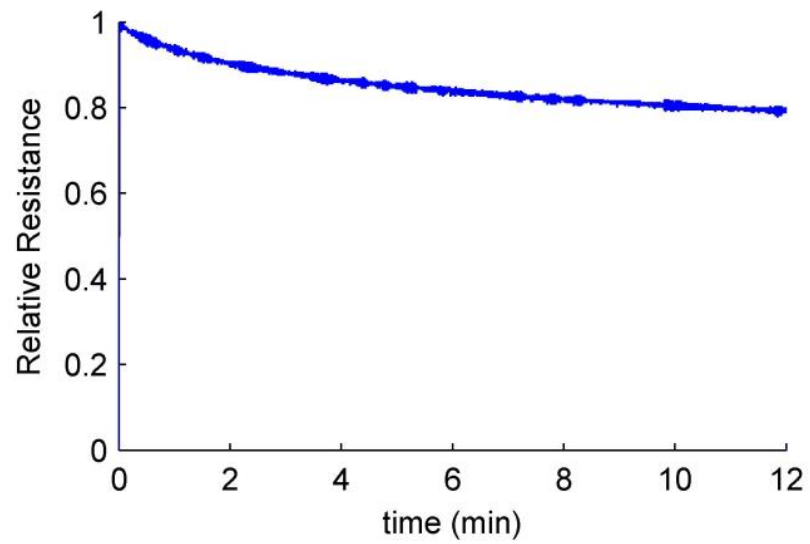

Fig. 8. Response of the stretch sensor under a maintained level of strain corresponding to $10 \%$. The minimum value of the relative resistance corresponds to the resistance of the unstrained sensor before applying the strain. The maximum value corresponds to the resistance of the sensor immediately after applying the level of strain to the sensor. also for both positive and negative strain rate (loading and unloading section), as well as the stability of the sensor response depending on the cycle number.

These results show that the relationship between the resistance and the sensor is of the third order in the unloading section. The gauge factor calculated in the loading section of the 60th cycle decreases by $8 \%$ with respect to that of the second cycle. The resistance corresponding to $0 \%$ of strain also decreases by $2 \%$ during the test. Finally, the third test revealed the drift of the resistance under a maintained level of strain. Indeed, when the strained is applied, the resistance decreases by $10 \%$ after two minutes, and by $21 \%$ after twelve minutes, as shown in Fig. 8. These results are in accordance with the results reported by Vipin et al. [22]. Like for polymer-based resistive sensors [23, 24], we can suppose that the resistance is affected by the stress relaxation of the materials and by the viscoelastic effects, mainly in the first cycles.

As shown by the test in the climatic chamber, the drift of resistance due to the environment temperature is lower than $\pm 1.5 \%$ with respect to the resistance at $25{ }^{\circ} \mathrm{C}$ in the range 10 ${ }^{\circ} \mathrm{C}$ to $40{ }^{\circ} \mathrm{C}$. We obtained similar results when the sensor was kept loaded at $10 \%$ inside the chamber in the same temperature range. Before starting the measurements, we waited $1 \mathrm{~h}$ in order to be sure that the resistance drift due to the deformation was negligible. Since the temperature of the environment and of the body are inside the investigated temperature range, we supposed negligible the effect of the temperature on the resistance drift with respect to the relaxation and loadingunloading effects.

\section{MEASUREMENT SYSTEM VALIDATION}

\section{A. Protocol}

Ten healthy subjects under the age of 37 were recruited as participants (genre, age and height are reported in Table II).

TABLE II

OVERVIEW OF THE TEN PARTICIPANTS' GENRE, AGE AND HEIGHT.

\begin{tabular}{cccc}
\hline Subject & Genre $^{\mathrm{a}}$ & Age & Height $(\mathrm{m})$ \\
\hline 1 & $\mathrm{~F}$ & 28 & 1.64 \\
2 & $\mathrm{M}$ & 37 & 1.81 \\
3 & $\mathrm{M}$ & 27 & 1.79 \\
4 & $\mathrm{~F}$ & 25 & 1.80 \\
5 & $\mathrm{M}$ & 28 & 1.74 \\
6 & $\mathrm{M}$ & 24 & 1.75 \\
7 & $\mathrm{M}$ & 22 & 1.80 \\
8 & $\mathrm{M}$ & 23 & 1.76 \\
9 & $\mathrm{M}$ & 23 & 1.78 \\
10 & $\mathrm{M}$ & 23 & 1.70 \\
\hline \multicolumn{4}{c}{}
\end{tabular}

The ten participants gave written, informed consent before inclusion in the study. None of them had impairments that would affect their gait during the experimental protocol.

We asked the participants to walk at their natural speed (usual pace) through a $25-\mathrm{m}$ long interior hallway for three times in both directions (six trials in total). Before starting the test, the subject was standing in the anatomical position: the body was standing erect, facing forward such as the feet. In this way, the back and the leg could be considered aligned. Besides wearing our measurement system on the left thigh, the subjects 
wore the XSens MTw Awinda system (XSens). It consisted of eight IMUs attached to the body through fast Velcro and body straps (Fig. 2). In detail, the IMUs were positioned on the thorax, pelvis, thigh, shank and foot of both legs. The eight sensors communicated wirelessly with a PC. We followed the tutorial and the suggestion of the company for the sensors placement, thus we used the system as wearable motion capture system for the lower limbs. According to its datasheet, the static and the dynamic accuracy is lower than $1.5^{\circ} \mathrm{rms}$. We adopted the software powered by NCS Lab for calculating the flexionextension of the hip. Before starting the tests, a calibration procedure for the XSens was required. We followed the calibration procedure suggested by NCS Lab [8]. This procedure ensures a worst-case standard error of $2.0^{\circ}$ for the hip angle. It was divided into two parts, called static and functional calibration. In the static calibration, the subject was asked to keep the standing position for five seconds; in this way, the software could define the anatomical coordinate system for thorax, pelvis, thigh, shank and foot. In the functional calibration, the subject was asked to stand in the upright posture and flex/extend each knee up to $70^{\circ}$ for five times; in this way, the software was able to define the anatomical coordinate system for the distal thigh.

The measurement data sent by all sensors were collected at $50 \mathrm{~Hz}$ by MVN Studio, provided by the Company. Since the sample rate of the XSens is lower than of our measurement system, we decreased the sample rate of our system to $50 \mathrm{~Hz}$.
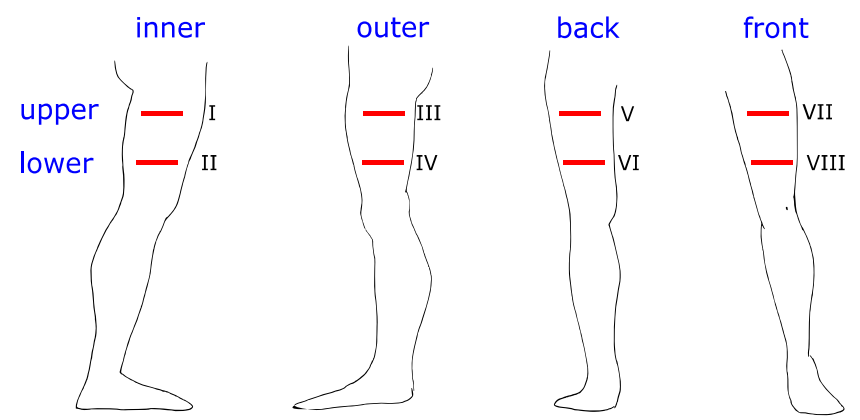

Fig. 9. The eight studied positions of the stretch sensor (red line) on the thigh.

The PC running at the same time MVN Studio and the custom VI designed for our measurement system was placed in the middle of the hallway against the wall due to the short wireless range indoor (about $20 \mathrm{~m}$ ) of XSens. Before starting the test, the subject tapped his left foot in order to synchronize the two measurement systems. We used the same protocol to evaluate the best positioning of the stretch sensor on the thigh. In particular, we put the sensor in the eight positions on the thigh: on the upper part of the posterior, anterior, inner thigh, and on the lower part of the posterior, anterior, inner and outer thigh.

\section{B. Results}

In the first part, we evaluated the best positioning of the stretch sensor on the thigh by selecting eight different positions (Fig. 9).

In the lower part of the thigh (II, IV, VI, and VIII), the elastic strap moved along the thigh during the tests, due to the anatomical shape of the leg; in the upper inner thigh (I) the
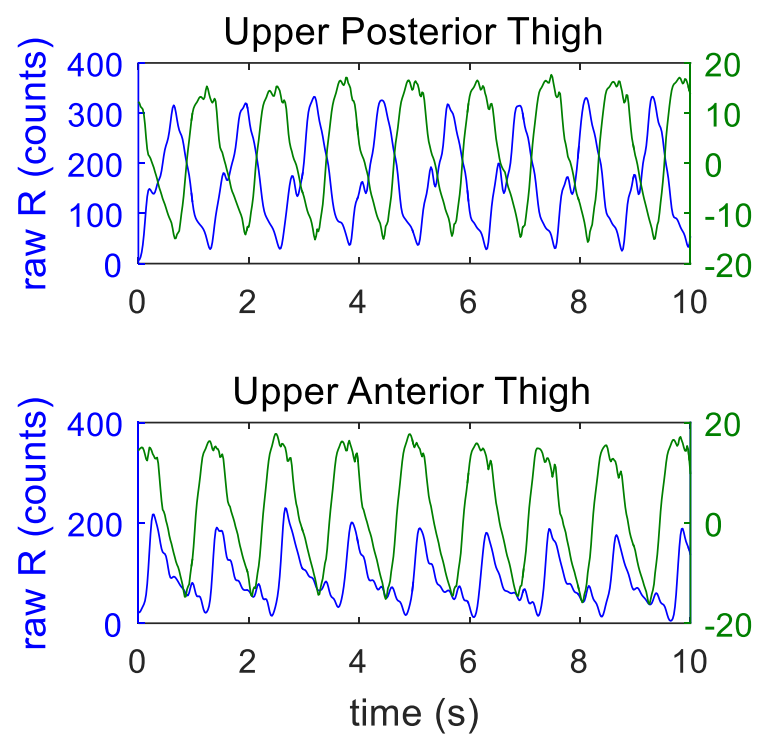

Fig. 10. Raw digital signal (blue line) from the stretch sensor when the sensor is put on the upper posterior thigh (upper panel) and on the upper anterior thigh (lower panel). Hip angle ( $\left.\alpha_{\mathrm{XSens}}\right)$ measured by the XSens (dotted green line). The measurements are referred to the subject 7 .

sensor scraped against the other thigh, whereas in the upper outer thigh (III) the response of the sensor was very low. The
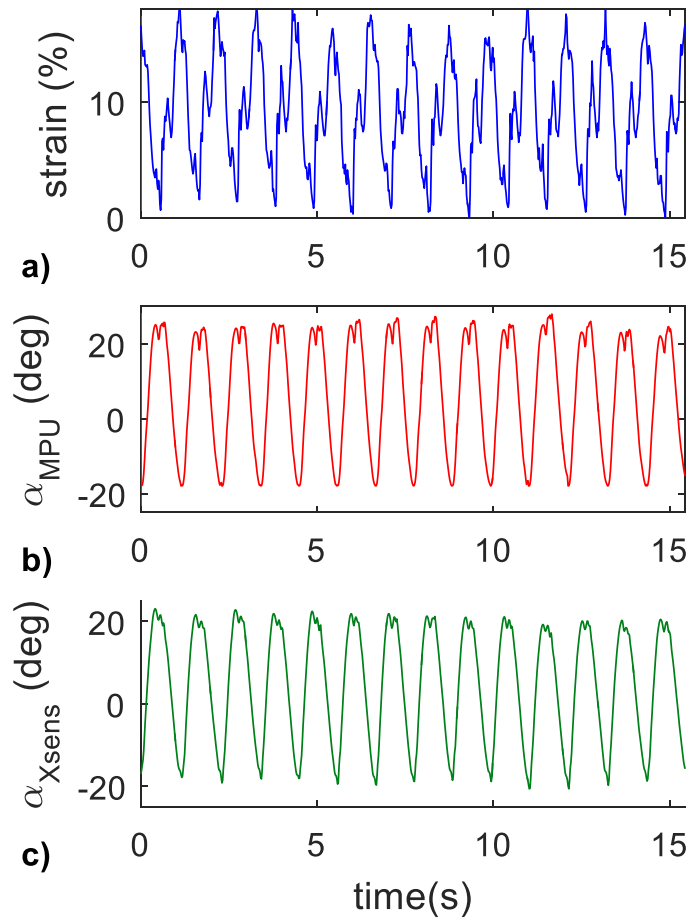

Fig. 11. Example of the measurements obtained from the three sensor systems during one trial (subject 4): a) the resulted strain calculating from the stretch sensor signal; b) joint angle of the hip calculated from the MPU 9150 sensor $\left.\left(\alpha_{\mathrm{MPU}}\right) ; \mathrm{c}\right)$ joint angle of the hip calculated from the XSens $\left(\alpha_{X S e n s}\right)$. 
sensor response in the two best positions (in the upper posterior and anterior thigh) is shown in Fig. 10. In detail, in the posterior thigh $(\mathrm{V})$ the response of the sensor is higher. The two peaks in each repetition suggest also a correlation between the stretch sensor strain and the muscle activity, since $\alpha_{x \text { Sens }}$ shows only one peak. For these reasons, we decided to put the sensor in the upper posterior thigh.

In Fig. 11, the signals obtained from the three different sensors in one trial for one subject (Subj 4) are shown as an example for maintaining a good readability of the figure. The trend of the three signals are comparable among the ten subjects. The signals are repetitive and suggest the possibility to use only one inertial sensor (MPU) to detect the flexion/extension of the hip. Since each subject performed the trials by walking at comfort speed, we also calculated the stride time from the three signals. The stride time is defined as the duration of one gait cycle; here we calculated the stride time (Table III) as the temporal distance between two local minimum points associated to two consecutive steps. The results of Table III prove the possibility to equally use the three type of sensors for the stride time calculation. Indeed, the average value is equal as well the standard deviation (SD). Although it is slightly higher for the stretch sensor case, SD can be considered negligible for all the sensors. The resulting walking speed varies from $1.05 \mathrm{~m} / \mathrm{s}$ to $1.5 \mathrm{~m} / \mathrm{s}$.

The angle of the hip joint tracked by the XSens, and by the $M P U$, as well as the strain of the stretch sensor are shown in Fig. 12. We reported the results of three subjects for comparison.

The angle tracked by $M P U$ was calculated according to the method described in section II, while the angle tracked by the XSens was calculated via NCS Lab software. Both calculated angles are in accordance with the typical trend reported in the literature [25]. The heel strike corresponds to $0 \%$ of the gait cycle.

In Fig.12c, there are three local maximum and one minimum points respectively at $20 \%, 50 \%, 90 \%$, and $35 \%$ of the gait
TABLE III

STRIDE TIME FOR EACH SUBJECT IN ONE TEST

\begin{tabular}{c|ccc} 
& \multicolumn{4}{|c}{ STRIDE TIME FOR EACH SUBJECT IN ONE TEST } \\
Average Stride Time & $\mathbf{3}$ SD $(\mathbf{s})$ \\
Subj & XSens & MPU & Stretch \\
\hline 1 & $1.11 \pm 0.03$ & $1.11 \pm 0.03$ & $1.11 \pm 0.05$ \\
2 & $1.29 \pm 0.03$ & $1.29 \pm 0.03$ & $1.29 \pm 0.03$ \\
3 & $1.25 \pm 0.05$ & $1.25 \pm 0.05$ & $1.25 \pm 0.07$ \\
4 & $1.21 \pm 0.01$ & $1.21 \pm 0.03$ & $1.21 \pm 0.07$ \\
5 & $1.23 \pm 0.03$ & $1.23 \pm 0.04$ & $1.23 \pm 0.05$ \\
6 & $1.23 \pm 0.03$ & $1.23 \pm 0.03$ & $1.23 \pm 0.04$ \\
7 & $1.14 \pm 0.04$ & $1.14 \pm 0.04$ & $1.14 \pm 0.05$ \\
8 & $1.19 \pm 0.05$ & $1.19 \pm 0.05$ & $1.20 \pm 0.05$ \\
9 & $1.25 \pm 0.04$ & $1.25 \pm 0.04$ & $1.25 \pm 0.04$ \\
10 & $1.56 \pm 0.07$ & $1.57 \pm 0.07$ & $1.56 \pm 0.09$ \\
\hline
\end{tabular}

The average stride time and one experimental standard deviation are calculated for each subject in one test (six trials) from the signals of the XSens system, $M P U$ sensor and stretch sensor.

cycle. As reported in [26, 27], the thigh muscles are activated mainly during the loading response, the pre-swing and terminal swing, whereas the thigh muscles are relaxed during the terminal stance (35\% of the gait cycle).

We used Bland-Altman plot to compare the two measurements techniques for the hip angle estimation. In Fig. 13, the measurements of the hip angle obtained by the XSens and the $M P U$ during the test on the subject 2 are reported as an example. The angle tracked by the $M P U$ is underestimated; indeed, the average difference of the methods is about $1^{\circ}$, and the maximum difference is lower than $5.5^{\circ}$. This error is expected due to the rotation of the pelvis during walking. Indeed, the $\alpha_{M P U}$ does not include the pelvic tilt [28]. We obtained comparable results for all the subjects.

The stretch sensor reveals a different behavior of the skin strain with respect to the hip angle.

We validated our system also during running, to evaluate if the different muscle activity of the thigh influence the output of the stretch sensor. An example of the acquired signals is shown in

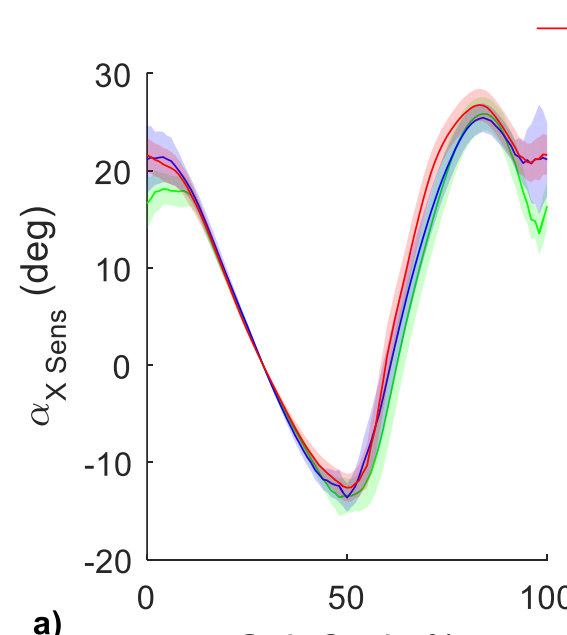

a) Gait Cycle \%

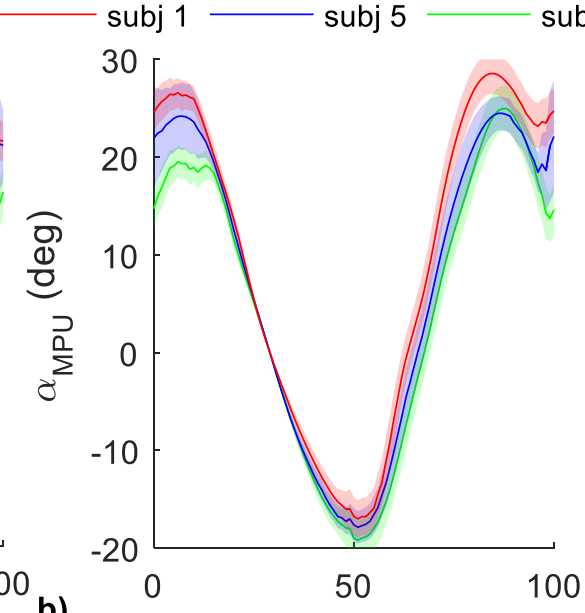

b)

Gait Cycle \%

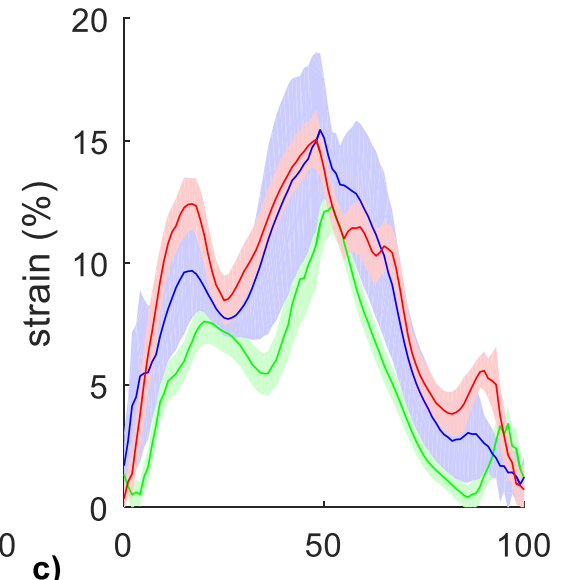

Gait Cycle \%

Fig. 12. Example of the angle of the hip joint of three subjects tracked by a) the XSens, b) the MPU and c) the strain of the stretch sensor. Each line is the mean and the shaded area indicates one standard deviation about the mean, calculated on a complete test (about 90 steps). A thicker line indicates greater variability in the signal. 


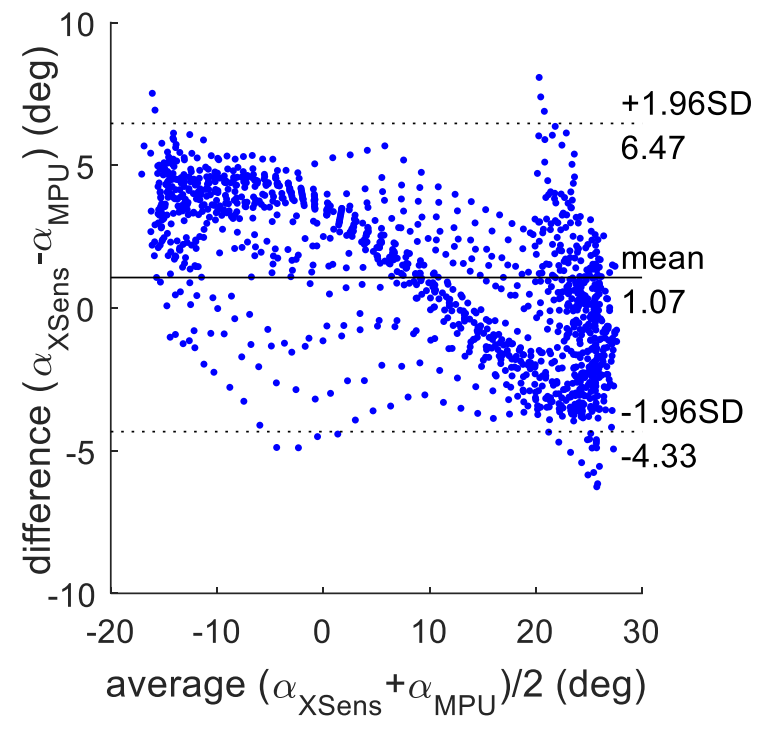

Fig. 13. Plot of differences between $X S$ Sens and $M P U$ vs. the average of the two measurements (subject 2). The line represents the difference average, while the dashed lines represent the limits of the $95 \%$ confidence interval.

Fig. 14. We verified that the trend of the three signals are comparable among the ten subjects.

In this test, the subject was asked to run freely along the hallway. The $\alpha_{M P U}$ tracked by the MPU device is comparable with the $\alpha_{X S e n s}$ tracked by the XSens, but it is different from the angle calculated during the walking test, as expected. The calculating running speed for subject 3 is $2.2 \mathrm{~m} / \mathrm{s}$.
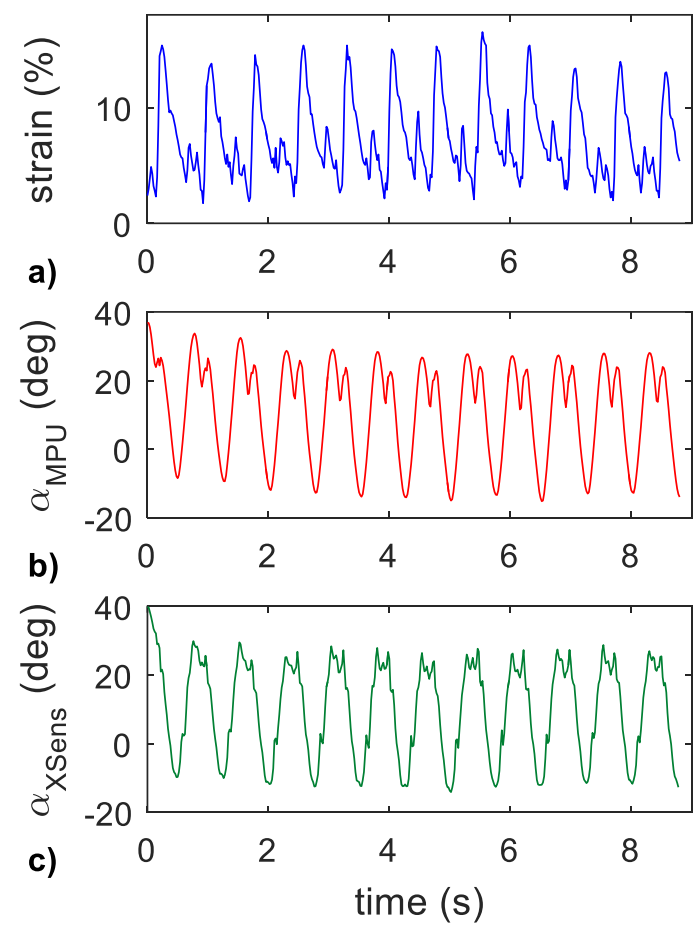

Fig. 14. Example of the measurements obtained from the three sensor systems during one trial (subject 3 ) during running: a) the resulted strain calculating from the stretch sensor signal; joint angle of the hip calculated from $b$ ) the MPU $9150\left(\alpha_{\text {MPU }}\right)$; c) joint angle of the hip calculated from the XSens $\left(\alpha_{X S e n s}\right)$.
Also the stretch sensor output has a different shape with respect to the walking test. The absolute maximum is located at $80 \%$ of the gait cycle, in the stance absorption phase. The different shape of the signal could have multiple reasons. First, the kinematic changes during running [29]; for example, it does not have the double support, as well as the stance phase is shorter. Also the ROM of the lower limbs differs with respect to the one during walking. Consequently, the muscle activity changes [29, 30]. The thigh muscles are much involved during running and for more time during one cycle for balancing the body and for increasing the speed and the ROM. In Fig. 14, a visible increment in strain is shown during running than during walking.

\section{CONCLUSIONS}

In this work, we present a new wearable and ease-to-use system for evaluating thigh movements during walking in a domestic environment. The system consists of an inertial sensor for measuring and a strain sensor for measuring the thigh muscle activity and its kinematic. The inertial sensor provides also the orientation of the thigh. Indeed, the deformation of the thigh skin onto the transverse plane of the thigh induces a strain in the sensor and thus the sensor resistance change. We evaluated the proposed system with a commercial system based on inertial devices considered as a reference instrument in the literature. The thigh inclination measured by the proposed system differs of maximum $5.5^{\circ}$ with respect to the hip angle measured by the commercial system. Furthermore, the strain sensor output can be related to the thigh muscle activity. This assumption has been sustained when the system is tested during walking and running.

The results proved that it is possible to use only one inertial sensor and one resistive sensor to measure kinematic and muscle activity. The system is ease-to-use and unobtrusive. It can easily be worn over adherent clothing. Wireless data transmission and long-life battery make the wearable system suitable for teleassistance and domestic applications, especially during the home therapy rehabilitation or for continuously monitoring lower limb musculoskeletal disorders and injuries. It can also be adopted during the physical activity indoor and outdoor. It is possible to easily interface the portable system with any mobile device (smartphone and tablet) because requires only a Bluetooth module. It is possible to easily integrate more sensors for detecting the movements of the other lower limb. Since the system is not directly in contact with the skin, washability did not be evaluated. Since the muscle physical strength influences the stretch sensor response during walking and running, in the future, the relationship between the stretch sensor and the muscle activity during walking will be investigated by using electromyographic and electrical impedance myography.

\section{REFERENCES}

[1] J. E. Graham, G. V. Ostir, S. R. Fisher, and K. J. Ottenbacher, "Assessing walking speed in clinical research: a systematic review," J. Eval. Clin. Pract., vol. 14, no. 4, pp. 552-562, 2008.

[2] B. C. Kieseier and C. Pozzilli, "Assessing walking disability in multiple sclerosis.," Mult. Scler., vol. 18, no. 7, pp. 914-924, 2012. 
[3] Wong, Z. Q. Zhang, B. Lo, and G. Z. Yang, "Wearable Sensing for Solid Biomechanics: A Review," IEEE Sensors Journal, vol. 15, no. 5. pp. 2747-2760, 2015.

[4] U. Jensen, P. Blank, P. Kugler and B. M. Eskofier, "Unobtrusive and Energy-Efficient Swimming Exercise Tracking Using On-Node Processing," IEEE Sensors Journal, vol. 16, no. 10, pp. 3972-3980, May15, 2016.

[5] I. H. López-Nava and A. Muñoz-Meléndez, "Wearable Inertial Sensors for Human Motion Analysis: A Review," in IEEE Sensors Journal, vol. 16, no. 22, pp. 7821-7834, Nov.15, 2016.

[6] P. Lorenzi, R. Rao, G. Romano, A. Kita and F. Irrera, "Mobile Devices for the Real-Time Detection of Specific Human Motion Disorders," IEEE Sensors Journal, vol. 16, no. 23, pp. 8220-8227, Dec.1, 2016.

[7] G. Orengo, A. Lagati and G. Saggio, "Modeling Wearable Bend Sensor Behavior for Human Motion Capture," IEEE Sensors Journal, vol. 14, no. 7, pp. 2307-2316, July 2014.

[8] A. G. Cutti, A. Ferrari, P. Garofalo, M. Raggi, A. Cappello, and A. Ferrari, "'Outwalk': A protocol for clinical gait analysis based on inertial and magnetic sensors," Med. Biol. Eng. Comput., vol. 48, no. 1, pp. 17-25, 2010.

[9] K. Lebel, P. Boissy, M. Hamel, and C. Duval, "Inertial measures of motion for clinical biomechanics: Comparative assessment of accuracy under controlled conditions - Effect of velocity," PLoS One, vol. 8, no. 11, 2013.

[10] M. Tesconi, A. Tognetti, E. P. Scilingo, G. Zupone, N. Carbonaro, D. De Rossi, E. Castellini, and M. Marella, "Wearable sensorized system for analyzing the lower limb movement during rowing activity," IEEE International Symposium on Industrial Electronics, 2007, pp. 2793-2796.

[11] Y. Menguc, Y.-L. Park, H. Pei, D. Vogt, P. M. Aubin, E. Winchell, L. Fluke, L. Stirling, R. J. Wood, and C. J. Walsh, "Wearable soft sensing suit for human gait measurement," Int. J. Rob. Res., vol. 33, no. 14, pp. 1748-1764, 2014.

[12] A. Tognetti, F. Lorussi, G. D. Mura, N. Carbonaro, M. Pacelli, R. Paradiso, D. De Rossi, and D. D. Rossi, "New generation of wearable goniometers for motion capture systems," J. Neuroeng. Rehabil., vol. 11, p. 56, 2014.

[13] A. Tognetti, F. Lorussi, N. Carbonaro, and D. de Rossi, "Wearable goniometer and accelerometer sensory fusion for knee joint angle measurement in daily life," Sensors (Switzerland), vol. 15, no. 11, pp. 28435-28455, 2015.

[14] S. J. Preece, L. P. J. Kenney, M. J. Major, T. Dias, E. Lay, and B. T. Fernandes, "Automatic identification of gait events using an instrumented sock.," J. Neuroeng. Rehabil., vol. 8, no. 1, p. 32, 2011.

[15] C. L. Lewis and S. A. Sahrmann, "Effect of posture on hip angles and moments during gait," Man. Ther., vol. 20, no. 1, pp. 176-182, 2015.
[16] R. Klotz, B. Colobert, M. Botino, and I. Permentiers, "Influence of different types of sockets on the range of motion of the hip joint by the transfemoral amputee," Ann. Phys. Rehabil. Med., vol. 54, no. 7, pp. 399410, 2011.

[17] M. Murray and S. Sepic, "Gait Patterns of Above-Knee Amputees Using Constant-Friction Knee Components," BPR, vol. 34, pp. 35-45, 1980.

[18] G. Hornero, D. Díaz, and O. Casas, "Bioimpedance system for monitoring muscle and cardiovascular activity in the stump of lower-limb amputees," Physiol. Meas., vol. 34, no. 2, pp. 189-201, 2013.

[19] J. Choi and K. Hong, "3D skin length deformation of lower body during knee joint flexion for the practical application of functional sportswear," Appl. Ergon., vol. 48, pp. 186-201, 2015.

[20] H. Lee, K. Hong, and Y. Lee, "Ergonomic mapping of skin deformation in dynamic postures to provide fundamental data for functional design lines of outdoor pants," Fibers Polym., vol. 14, no. 12, pp. 2197-2201, 2013.

[21] S. Chen, J. Lach, B. Lo and G. Z. Yang, "Toward Pervasive Gait Analysis with Wearable Sensors: A Systematic Review," in IEEE Journal of Biomedical and Health Informatics, vol. 20, no. 6, pp. 1521-1537, Nov. 2016.

[22] V. S. Vibhute and A. Kshirsagar, "Identification of Hysteresis And Relaxation Parameters in Stretch Sensor TM," no. 2, pp. 15-22, 2012.

[23] M. Borghetti, E. Sardini, and M. Serpelloni, "Evaluation of bend sensors for limb motion monitoring," in IEEE MeMeA 2014 - IEEE International Symposium on Medical Measurements and Applications, Proceedings, 2014.

[24] M. Borghetti, M. Serpelloni, E. Sardini, and S. Pandini, "Mechanical behavior of strain sensors based on PEDOT:PSS and silver nanoparticles inks deposited on polymer substrate by inkjet printing," Sensors Actuators, A Phys., vol. 243, pp. 71-80, 2016.

[25] Neumann, Kinesiology of the musculoskeletal system: foundations for rehabilitation," 2010.

[26] A. Strazza, A. Mengarelli, S. Fioretti, L. Burattini, V. Agostini, M. Knaflitz, F. Di Nardo, "Surface-EMG analysis for the quantification of thigh muscle dynamic co-contractions during normal gait", Gait \& Posture, Volume 51, January 2017.

[27] Vaughan, B. Davis, and J. O’Connor, Dynamics of human gait (Second Edition). Cape Town, South Africa: Kiboho Publishers, 1999.

[28] S. McIntosh, K. T. Beatty, L. N. Dwan, and D. R. Vickers, "Gait dynamics on an inclined walkway," J. Biomech., vol. 39, no. 13, pp. 2491-2502, 2006.

[29] N. Tom and T. Novacheck, "Review paper: The biomechanics of running," Gait Posture, vol. 7, pp. 77-95, 1998.

[30] R. A. Mann and J. Hagy, "Biomechanics of Walking, running and sprinting," Am. J. Sports Med., vol. 8, no. 5, pp. 345-350, 1980. 